\title{
A Comprehensive Study of the Behavior of Different TCP Variants using Multistate Error Model in Cellular Mobile Environment
}

\author{
S. M. Hasan Sazzad \\ lqbal \\ Pabna Science \& \\ Technology University, \\ Pabna, Bangladesh.
}

\author{
Md. Amran Siddiqui \\ Stamford University \\ Bangladesh, Dhaka, \\ Bangladesh
}

\author{
Shaiful Alam \\ Chowdhury \\ Stamford University \\ Bangladesh, Dhaka, \\ Bangladesh
}

\author{
Md. Rounok Salehin \\ Stamford University \\ Bangladesh, Dhaka, \\ Bangladesh
}

\begin{abstract}
The widespread use of internet services over the wireless links is expeditiously grown in recent years. The reinforcement of portable computing platform and technological elevation of wireless communication evokes substantial prosperity in the design and development of integrated environment such as cellular mobile environments. The repercussion of packet losses due to corruption and mobility is the radical circumstances of deteriorating TCP performance in the wireless ambience. Uniform and Multistate error model are both used in wireless environment; Uniform error model provides packet losses at a constant rate which consequence an imprecise results during simulation as in wireless environment packet losses are arbitrary, bursty and time diversify in nature. However Multistate error model imitates the behavior of the wireless packet loss in real environment and produce infallible outcome. In this paper we constitute a realistic Cellular Mobile Environment by considering multistage error model in the design of wireless packet losses. Mobility in structure is also conceived to estimate the actual performance of TCP. Moreover the behavior of TCP Tahoe, Reno, New Reno, Sack and Vegas in Cellular Mobile network is simulated to perceive the impact of wireless link on the behavior of these TCP variants. Finally from the result of our simulation we conclude the best TCP variants for different circumstances.
\end{abstract}

Keywords: Multistage Error Model, Simulation Scenario, High Bit error, handoff and speed.

\section{INTRODUCTION}

The mobile wireless net and data net is evolved independently in the earlier stage of their invention. However with time it becomes necessary to develop an integrated platform of mobile net and data net for consummating the user demand for miscellaneous services [2]. The second generation (2G) wireless nets were only appropriated for the communication of voice traffic. Moreover the security was a big concern in $2 \mathrm{G}$ wireless system and along with time it also fails to effectuate the user demand. Eventually the third generation and emerging forth generation wireless system provides reliable data services over wireless links using handy and portable devices such as mobile phone, laptop etc[10]. Transmission Control Protocol (TCP) [8] is an widely used transport layer protocol for internet which performs excellently for wired nets when initially designed. However the traditional TCP fails to reflect several challenges for transmitting data over wireless nets such as data over high and time varying bit error rate, variable delay, mobility (handoff) and interaction with lower layer protocols [1]. In wired link only packet losses due to congestion is considered during inference of congestion control whereas wireless link errors (random packet loss) and mobility are mostly responsible for packet looses in wireless links [9]. According to the design specification of the TCP's congestion control algorithm, TCP is unable to discriminate the loss caused by the three congestion control factors congestion, wireless link error or mobility.

\section{TCP IN WIRELESS ENVIRONMENT}

In recent years there is a strong interest in broadening the Internet access technologies to wireless and satellite links. There are many advantages in using these technologies. This technological advancement provides several benefits such as enable user mobility and net access anytime from anywhere, enhance data rate etc [7]. When using wireless links it needs to be considered that they have quite different characteristics to wired links; they can have a high bit error rate (BER), often combined with fading, can be asymmetric in nature (different forward and return channel bandwidth) or have a high propagation delay etc [5]. Not all of these characteristics might be present in any given link but all of them, or even a subset of them, pose difficulties to the traditional TCP protocol. There are several characteristics that are unique to the wireless environment that make it challenging to adapt TCP to effectively in this environment. The major factors affecting TCP performance in cellular mobile environment are limited bandwidth, long round trip times for either slow congestion window growth or longer time to detect and recover from losses, random losses, user mobility etc [4].

\section{SIMULATION MODEL AND PARAMETERS}

The simulation model (net topology) used in the simulations is shown in figure 1. As shown in figure, there are five nodes: two fixed host (FH): Node1 and Node2, two base stations (BS), Base station1 and Base station2, and a Mobile station (MS). Each base station includes a finite buffer drop-tail gateway. In this research only bulk data transfer are considered from Node1 to mobile station. Furthermore FTP is used as an application layer protocol. It is assumed that TCP segments are transmitted towards the wireless terminals from the wired terminal, and ACK segments are in the reverse direction. It is also considered that packet loss is mainly caused by the transmission error on the wireless link. A Multistate error model is implemented in wireless link. This error 
model has two states. They are good state and bad state. The transition from one state to another state depends on a matrix that is given below,

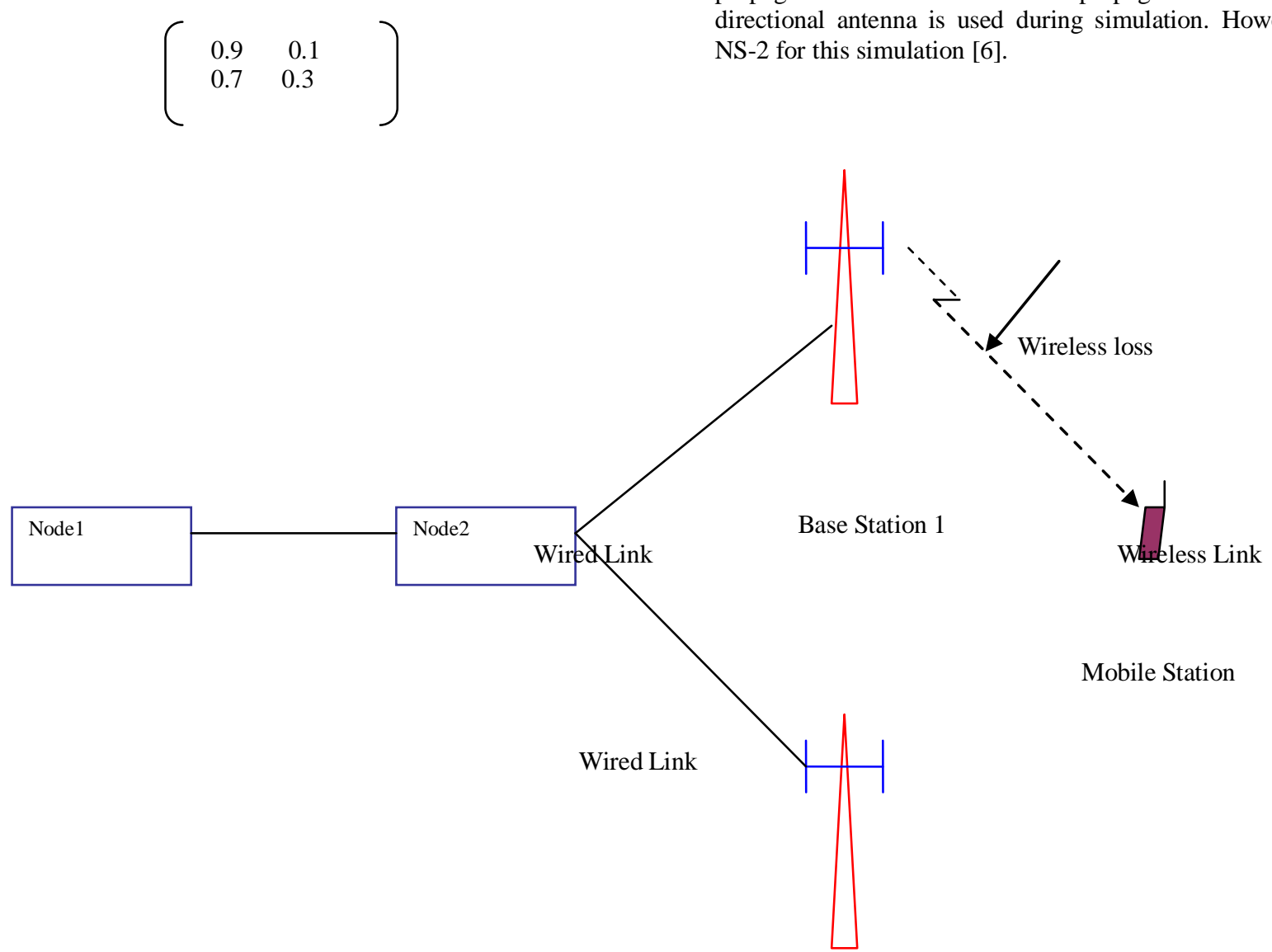

Base Station 2
The occurrences of these two states have the same probability and which state comes first that is also random and this process continues for the whole simulation time. Here two ray ground propagation model is used as a propagation model and Omni directional antenna is used during simulation. However we use NS-2 for this simulation [6]

Fig 1: Simulation Scenario 
The topology boundary is defined as 500 X 500 and the mobile station is allowed to move within this boundary, traversing from the coverage of one base station to the coverage of another base station at a specified speed. MobileIP is used in order to perform handoff between the two base stations. We use the IEEE 802.11 MAC layer for the wireless net. This choice is based on the availability in the NS-2 simulator and because most of the research that we refer to in the research uses it. Table 1 shows the list of simulation parameters,

Table 1: The Simulation Parameters

\begin{tabular}{|c|c|c|}
\hline & & \\
\hline Wired link capacity & \multicolumn{2}{|c|}{$10 \mathrm{Mbps}$} \\
\hline Wired propagation delay & \multicolumn{2}{|c|}{$20 \mathrm{~ms}$} \\
\hline Wireless link capacity & \multicolumn{2}{|c|}{$2 \mathrm{Mbps}$} \\
\hline Wireless propagation delay & \multicolumn{2}{|c|}{$2 \mathrm{~ms}$} \\
\hline TCP packet size & \multicolumn{2}{|c|}{1040 byte } \\
\hline TCP ack packet size & \multicolumn{2}{|c|}{40 byte } \\
\hline Buffer size of each base station & \multicolumn{2}{|c|}{50 packets } \\
\hline Coverage of each base station & \multicolumn{2}{|c|}{250 meters } \\
\hline Speed of the Mobile station & \multicolumn{2}{|c|}{$\begin{array}{c}0 \text { to } 50 \\
\text { meter/second }\end{array}$} \\
\hline \multirow[b]{2}{*}{ Wireless packet loss rate } & $\begin{array}{l}\text { Good } \\
\text { state }\end{array}$ & 0 \\
\hline & $\begin{array}{l}\text { Bad } \\
\text { state }\end{array}$ & $\begin{array}{c}0.0001 \\
\text { to } \\
0.1\end{array}$ \\
\hline
\end{tabular}

To evaluate the performance of the different TCP clones in wiredcum-wireless environment, the following performance metrics are used:

$>$ Packet Error rate: The packet error rates on the wireless link.

$>$ Number of Handoffs: The number of times the mobile station moves from the coverage of one base station to another base station.

$>$ Speed: The speed at which the mobile station moves. Setting these parameters, we measure the throughput (the amount of data the receiver receives with out retransmission in a unit time) and also see the congestion window dynamics of different TCP clones to analyze their behavior in the presence of multistate error in cellular mobile environment.

\section{SIMULATION RESULTS AND DISCUSSION}

\subsection{Effect of high bit error rate}

Simulations are performed to investigate the performance of TCP variants in the presence of multistate error model. The good state has the 0 wireless packet error rate, i.e. no packet is lost in the good state and the bad state has the wireless packet error rate ranging from $10^{-4}$ to $10^{-1}$. Each simulation is performed for 200 seconds. From Figure 2 it can be observed that TCP throughput degrades with increasing packet error rate that proves its poor performance. Throughput starts to decrease even at low packet error rate such as 0.001 . Since TCP's congestion algorithm is responsible for TCP throughput, it leads to poor performance in high error rate environment, because it treats the packet losses due to wireless error as a symbol of net congestion and consequently reduces the congestion window. Figure 2 also compares five mentioned TCP variants. In terms of throughput, the implementations can be ordered as Sack followed by NewReno, then Reno, then Tahoe and finally Vegas.

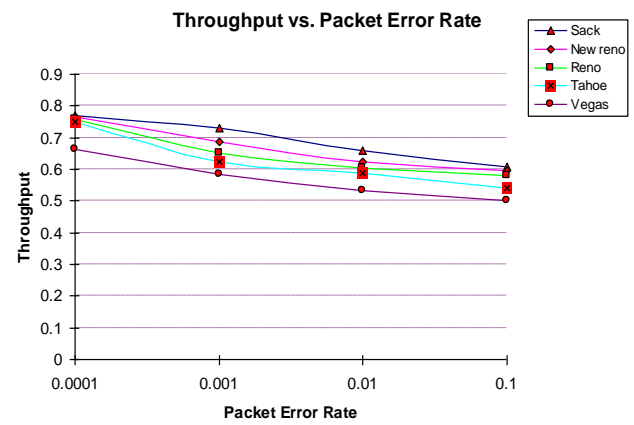

Fig. 2: Throughput Comparison of TCP Tahoe, Reno, NewReno, Sack and Vegas over cellular mobile network for PER ranging from $10^{-4}$ to $10^{-1}$ without considering handoff

Figure 3 shows the plots of the congestion window for all TCP variants. The values of cwnd are taken from a simulation runtime of 200 seconds with the presence of packet error rate of $10^{-3}$. However for better understanding of the behavior of congestion window of each TCP variants, the value of the congestion window is traced for an interval of every second. When comparing the plots, it can be noticed that the Tahoe plot shown in Figure 3(a) has the largest number of occurrence of cwnd $=1$ values. This is because Tahoe TCP slow-starts every time a packet is dropped. This caused Tahoe TCP to have poor average congestion window and the poor performance whereas TCP Sack has the best average congestion window value as well as the best performance.

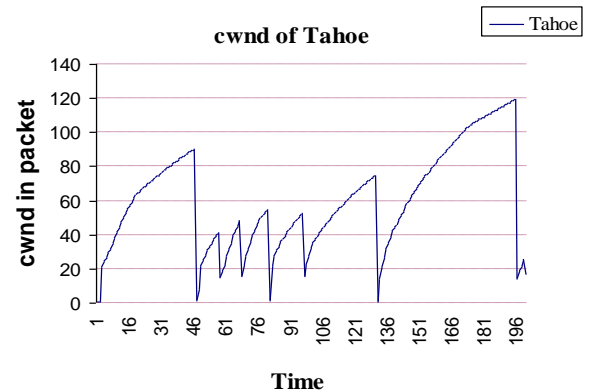

Fig 3(a): Congestion window dynamics of TCP Tahoe

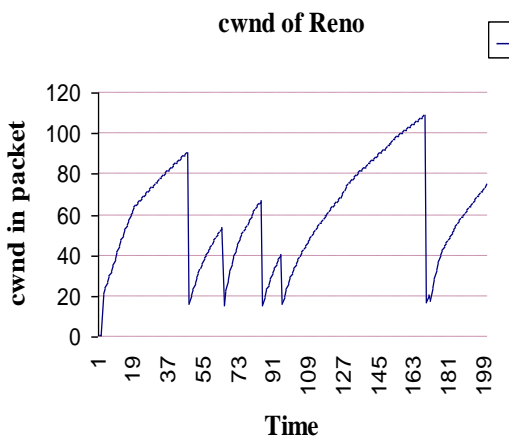

Fig 3(b): Congestion window dynamics of TCP Reno 


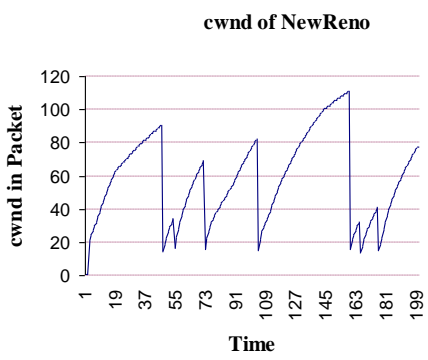

Fig 3(c): Congestion window dynamics of TCP NewReno

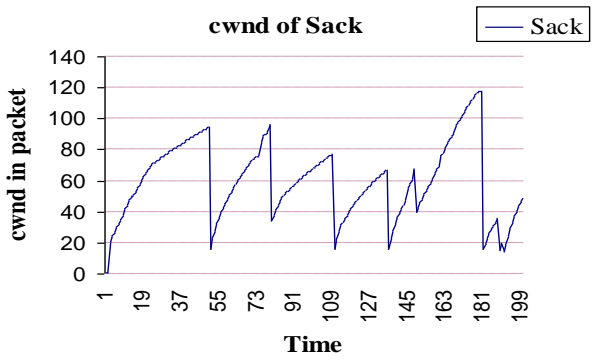

Fig 3(d): Congestion window dynamics of TCP Sack

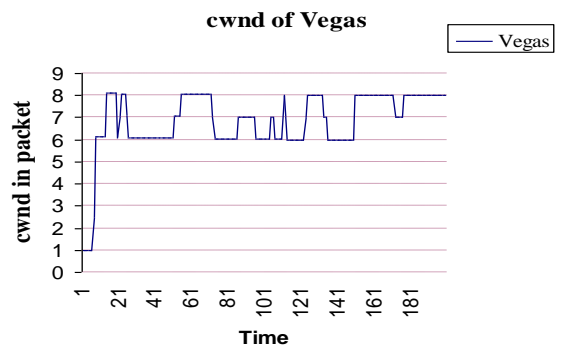

Fig 3(e): Congestion window dynamics of TCP Vegas

From the Figure 3(e), it is noticed that TCP Vegas has the least congestion window growth and for this reason it performs bad in the cellular mobile environment. Figure 4 shows how the Congestion window of TCP reacts with increasing error rate. It shows that as the packet error rate increases, TCP congestion window fluctuates more rapidly and thus gets less opportunity to grow its size, which results poor throughput.

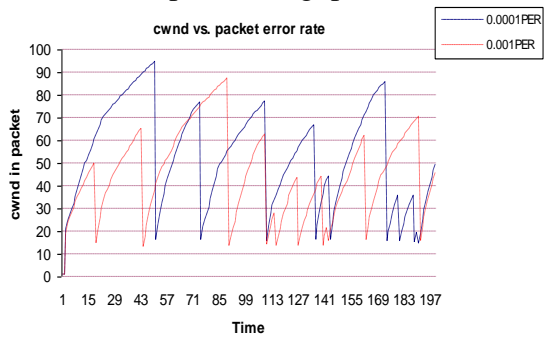

Fig 4: Congestion window dynamics of TCP Sack over cellular mobile network for PER 0.0001 and 0.01

\subsection{Effect of handoff and speed}

The handoff event in the wireless net is mainly happened due to mobility of the mobile station. Hence simulation is performed to analyze the effect of mobile station's speed with incorporated handoff on the performance of TCP. The speed of mobile station is varied from 0 meter/second to 50 meter/second. And the simulation is run for 200 seconds. Here we only consider the unmodified TCP. The result is plotted in Figure 5. From the figure 5 it can be shown that throughput has been severely degraded with increasing speed.

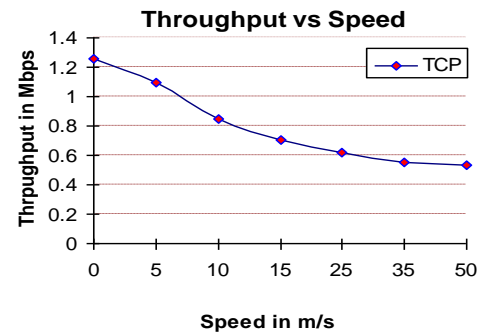

Fig 5: Shows the graph of throughput vs. speed of the mobile station exploring the performance of TCP during the mobility of mobile station

Simulation is also carried out to investigate the behavior of regular TCP in presence of mobile handoff. To simulate the handoff performance on TCP, four handoffs are generated.. For each handoff the mobile station shifts from the Home Agent (Base Station1 of Figure 1) to the Foreign Agent (Base Station2 of Figure 1) and vice versa. Figure 6 shows the Congestion window dynamics for four handoffs. It can be shown that whenever a handoff occurs congestion window is reduced to its minimum value, i.e., cwnd $=1$.

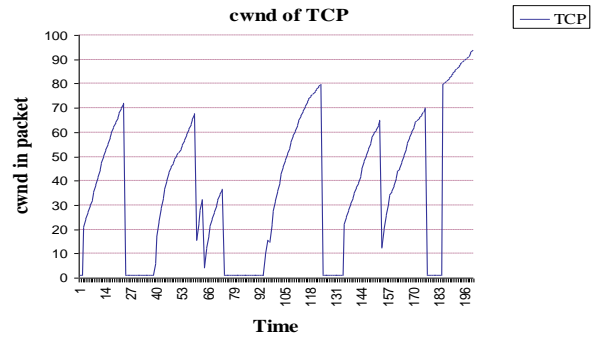

Fig 6: Congestion window dynamics of unmodified TCP in presence of mobile Handoff (Speed $10 \mathrm{~m} / \mathrm{sec}$ )

\subsection{Effect of high bit error rate and handoff}

Simulation is also carried out to analyze the performance of five TCP variants in the presence of both packet error rate and handoff. Packet error rates are varied from $10^{-4}$ to $10^{-1}$ for the bad state and 0 for the good state. Four handoffs are generated and the simulation is run for 200 seconds. The result is plotted in Fig 7.

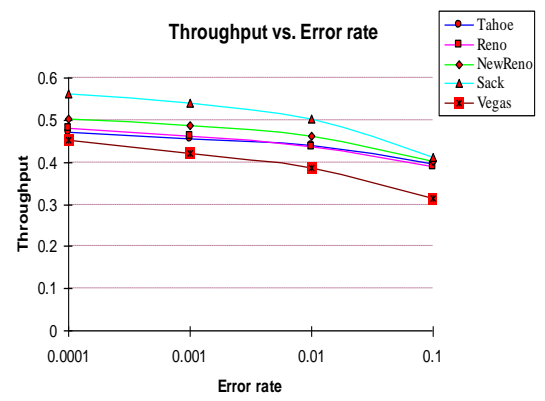

Fig 7: Throughput Comparison of TCP Tahoe, Reno, NewReno, Sack and Vegas over cellular mobile network for PER ranging from $10^{-4}$ to $10^{-1}$ considering handoff 
From the figure 7 it is observed that throughput has been severely degraded in the presence of packet error rate and handoff because a large number of packets has been lost due to handoff and packet error rate. From the figure 7 it is also noticed that TCP Sack has the best performance and TCP Vegas the worst performance.

\section{CONCLUSION AND FUTURE WORK}

In this thesis we have took a close look on the two important characteristics of the wireless network, high bit error rate of the wireless link and mobility and their effect on TCP performance. To model the high bit error of the wireless link, we have used the multistate error model which gives the more accurate and adequate representation of the wireless losses. As we know, in wireless link the losses are random and varying in nature, so these losses can not be accurately modeled by the uniform error model, where packet is lost at a uniform rate during the whole simulation. But in multistate error model, there are two states. One is good state and another is bad state. Good state is represented by the zero loss probability and the bad state is represented by high loss probability and these two states occur repeatedly throughout the whole simulation, which mimic the wireless loss behavior more preciously. In this mobility is considered by generating handoffs when a mobile station switches from its serving base station (Home agent) to another base station (Foreign agent) and viceversa. We have also considered the speed of the mobile station, where the mobile station moves freely at different speed at different times through out the whole area that is considered in this research. From the simulation it is mentioned that the higher the bit error rate, the less the throughput of TCP. In this research, the performance of five different clones of TCP (Tahoe, Reno, NewReno, Sack, Vegas) have been analyzed in the presence of high bit error. From the analysis it can be shown that TCP Sack has a superior performance than the other TCP clones those are considered in this research because it has the ability to indicate multiple packet losses from the same window and also has a selective acknowledgement mechanism to combat the problems caused by this multiple packet losses. TCP Vegas has the worst performance among all the considered TCP clones. Also the effect of mobility (with incorporated Handoff) on the performance of TCP has been simulated. From the analysis, it is seen that the throughput of TCP degrades with increasing the speed of the mobile station. We have also analyzed the behavior of these five TCP clones in the presence of both high bit error and handoff. From the analysis, it is observed that the throughput of these five TCP clones seriously degrades because of heavy packet losses due to both handoffs and high bit error and it is also mentioned that Sack has the best performance.

There are several key areas for extension of our current research.

The first important area is to simulate two types fading, such as small scale fading (Multipath delay spread and Doppler Effect) and the large scale fading (Shadowing effect) that are normally found in mobile cellular environment and also analyze their effect on the performance of TCP.

The second is to analyze behavior of TCP in cellular mobile environment considering coverage, battery power, and other impacts that are found in cellular mobile environment.

\section{REFERENCES}

[1] Ns_simulations_shops_mo_aug_2005.pdf http://wine.icu.ac.kr

[2] NTT. DoCoMo subscriber growth, Jan, 2004 http://www.nttdocomo.com/companyinfo/subscriber.html

[3] [3]. Van Jacobson, "Congestion Avoidance and Control," in Proceedings of ACM SIGCOMM '88, Aug. 1988, pp. 314329.

[4] K. Pentikousis , "TCP in wired-cum-wireless environments," IEEE Communications Surveys \& Tutorials, vol. 3, no. 4, Fourth Quarter 2000.

[5] G. Xylomenos, G.C.Polyzos, P.Mahonen, and M.Saaranen, "TCP Performance Issues over Wireless Links", IEEE Communications Magazine, Apr. 2001.

[6] NS, The net simulator - ns-2., URL:http://www.isi.edu/nsnan/ns

[7] Hassan, M. and Jain, R. (2001), TCP Performance in Future Networking Environments. Guest Editorial, IEEE Communications Magazine [Online], April 2001, pp. 51. http://www.cis.ohio-state.edu/ jain/papers/tcp_ed.htm

[8] J. Postel, "Transmission Control Protocol," RFC 793, Sept.1981.

[9] V. Tsaoussidis and I. Matta. Open issues on TCP for mobile computing. Journal of Wireless Communications and Mobile Computing, 2(1):3-20, Feb. 2002.

[10] T. S. Rappaport. Wireless communications, principles and practice. Prentice Hall, 1996. 